Kompass

Dermatologie

\title{
Aus der Praxis
}

\section{Die Praxis der dermatologischen Lasermedizin}

Die Dermatologie mit ihren medizinischen und kosmetischen Indikationen bietet von allen Fachgebieten die größte Vielfalt an einsetzbaren Lasertypen. Folgende biophysikalische Wirkprinzipien sind hilfreich zum Verständnis der Gewebe-Licht-Wechselwirkungen:

Die thermische Wirkung ist unspezifisch und wirkt gleichermaßen auf alle Gewebeanteile, auf die sie trifft. Die selektive Photothermolyse beschreibt die Beschränkung der Wirkung eines Laserstrahls auf ein bestimmtes Zielchromophor. Durch die Wahl einer spezifischen, vom Zielchromophor gut absorbierten Wellenlänge, wird die Umgebung geschont. Die thermokinetische Selektivität beschreibt die Konzentration der Laserenergie in einer Zielstruktur durch die Wahl einer an deren thermische Relaxationszeit (Zeit bis zur Abkühlung auf die Hälfte einer bestimmten Maximaltemperatur) angepassten Impulsdauer.

Angelehnt an diese Prinzipien werden nachfolgend die wesentlichen Lasertypen dargestellt und zusammen mit ihren Indikationen erläutert.

\section{Gefäßlaser}

Um vaskuläre Veränderungen mittels Lasers zu therapieren, muss sich die Impulsdauer an der Größe der Gefäße orientieren. Typischerweise liegt die Impulsdauer im Bereich von 0,5-100 Millisekunden. Die Wellenlänge sollte idealerweise in der Nähe des Absorptionsmaximums des oxygenierten Hämoglobins liegen (560$590 \mathrm{~nm}$ ). Besonders gut eignen sich hier der gepulste Farbstofflaser (585/595nm) und der KTP-Nd:YAG-Laser (532nm). Für tiefere Gefäße muss der Nd:YAG-Laser (1064nm) verwendet werden, da die Eindringtiefe der erstgenannten Systeme nicht groß genug ist. Typische Indikationen sind Teleangiektasien, Angiome, Feuermale, Besenreiser (mit Einschränkungen).

\section{Pigmentlaser}

Zielchromophoren sind hier kleine Pigmentpartikel (Tattoos oder natürliche Hyperpigmentierungen) mit sehr kurzen thermischen Relaxationszeiten. Daher sind Impulse im Nano- oder Picosekundenbereich notwendig, um die Strukturen unter Schonung der Umgebung zu zerstören. Die notwendige Wellenlänge orientiert sich an der Farbe der Pigmente. Nachfolgend die für typische Pigmentfarben passenden Lasertypen und die dazugehörigen Wellenlängen:

- Schwarz: Nd:YAG (1064nm), Alexandrit (755nm), Rubin (694nm)

- Grün/Blau: Alexandrit (755nm), Rubin (694nm)

- Braun: Alexandrit (755nm), Rubin (694nm), KTP-Nd:YAG (532nm)

- Rot: KTP-Nd:YAG (532nm)

Weiße und gelbliche Pigmente lassen sich kaum entfernen. Bei der Behandlung von Permanent-Makeup kann ein Farbumschlag durch die chemische Alteration der Pigmente entstehen.

\section{Ablative Laser}

Das Zielchromophor hierbei ist Wasser, welches einen sehr hohen Absorptionskoeffizienten für das Licht des CO2-Lasers (10600nm) und sogar eine Größenordnung mehr für das Licht des Erbium:YAGLasers (2940nm) hat. Durch die starke Absorption dringt die Laserenergie nicht tief ein, sondern wird bereits in den obersten Schichten der Epidermis/Dermis absorbiert und führt zu einer Absprengung dünner Schichten im Mikrometerbereich. Beim CO2-Laser ergibt sich ein Wärmeeintrag in tiefere Regionen, die thermische Effekte auf Kollagenfasern ausüben. Beim Erbium:YAG-Laser entsteht fast keine Wärme, man spricht auch von kalter Ablation.

Die Indikationen sind sehr vielfältig und reichen von der Entfernung benigner Tumoren, der Behandlung von Narben bis hin zur Faltenstraffung und Glättung von Aknenarben. Aktuelle Entwicklungen sind die «Laser assisted drug delivery» und die adjuvante Anwendung vor der Behandlung von Tattoos, um die Ausschleusung zu verbessern.

\section{Photoepilationslaser}

Dies ist der Indikationsbereich mit den wahrscheinlich höchsten Behandlungsanzahlen. Zielchromophor ist das Melanin der Haare. Daraus folgt, dass helle oder blonde Haare nicht photoepiliert werden können. Die Impulsdauern liegen meist im Bereich 5-100 Millisekunden. Geeignete Lasergeräte sind, je nach Hauttyp, der Alexandritlaser (755nm) für helle Haut, Diodenlaser (800-900nm) für dunklere Haut und der Nd:YAG-Laser (1064nm), mit dem auch der Hauttyp VI behandelt werden kann.

\section{Sicherheit}

Bei falscher Anwendung von Lasern gibt es vielfältige, zum Teil schwere Nebenwirkungen, weshalb die Notwendigkeit einer fundierten Ausbildung nicht genug betont werden kann. Dies ist unter anderem möglich bei der Deutschen Dermatologischen Lasergesellschaft (www.ddl.de) oder in dem universitären Aufbaustudiengang «Diploma in Aesthetic Laser Medicine» der Universität Greifswald (www.laserstudium.com).

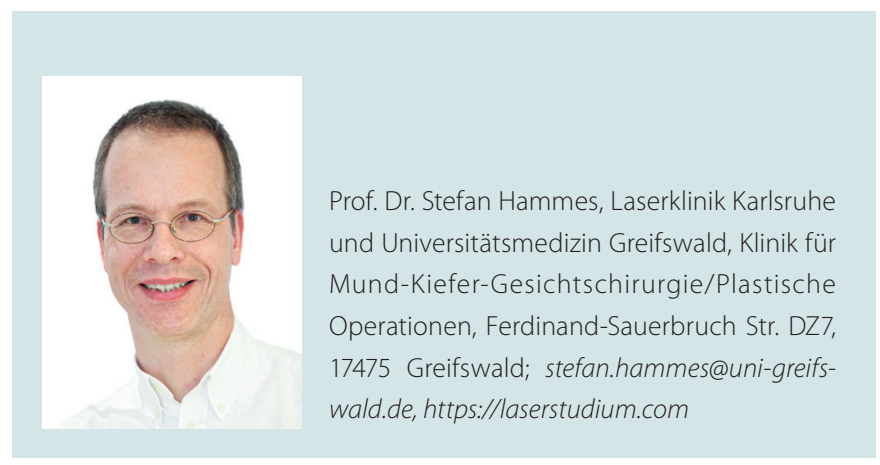




\section{Bei Facharztweiterbildung in der Niederlassung}

Für Johanna Dörner, die gerade mit der Weiterbildung begonnen hat, und ihre Schwester Dr. Friederike Dörner, die 2017 ihre Weiterbildung abgeschlossen hat, war es klar: sie absolvieren ihre Facharztweiterbildung in der Niederlassung und zwar in der Praxis ihres Vaters Dr. Martin Dörner. Eine spannende Konstellation in die sie uns im folgenden Interview einen tiefen Einblick gewähren.

\section{Welche Beweggründe hatten Sie, einen Teil Ihrer Facharzt- weiterbildung bei einem niedergelassenen Facharzt zu machen?}

Johanna Dörner (JD): Für den Start in meine Assistenzarzttätigkeit hätte ich mir kein besseres Arbeitsumfeld denken können. Die Bedingungen in der Praxis sind super. Das Spektrum der Behandlungsmöglichkeiten ist ähnlich dem im stationären Bereich.

Unter Anleitung meines Vaters und meiner Schwester lerne ich viel. Ich fühle mich in der Praxis sehr wohl, schließlich bin ich hier aufgewachsen.

Friederike Dörner (FD): Auch ich habe meine Facharztweiterbildung in der Kassenarztpraxis meines Vaters begonnen. Dort konnte ich die verschiedenen konservativen wie operativen Bereiche der Augenheilkunde kennenlernen. Nach zwei Jahren habe ich in die Augenklink Köln-Merheim unter der Leitung von Prof. Dr. Dr. hc. Norbert Schrage gewechselt. Jede der beiden Ausbildungsstellen hat ihre Besonderheiten.

\section{Welche Vor- bzw. Nachteile an der Facharztweiterbildung in der Niederlassung sehen Sie?}

JD: Viele angehende Augenärzte ziehen eine Weiterbildung in der Praxis nicht in Betracht. Die Klinik ist ihnen aus dem Studium bereits bekannt. Sie kennen die Hierarchien und Arbeitsstrukturen. Die Verantwortung für die Patienten verteilt sich auf die gesamte Abteilung.
FD: In der Praxis arbeitet man sehr selbstständig. Innerhalb kürzester Zeit müssen effektiv Patienten untersucht, Befunde erhoben und viele Entscheidungen getroffen werden. Das Spektrum der Erkrankungen ist in der Basisversorgung groß. Kommt fachlich einmal eine Unklarheit auf, so ist der Chef immer in greifbarer Nähe. Die meisten Fragen klären sich im Handumdrehen, wenn der Chef erst einen Blick durch die Spaltlampe geworfen hat.

Wir erleben die Weiterbildung in der Praxis als positiv. Wir sind mit 4 Weiterbildungsassistentinnen und 2 Fachärzten ein kleines Team, in dem sich alle mit Rat und Tat zur Seite stehen. [...]. Kurze Wege und gute Organisation vereinfachen die Abläufe in der Praxis. Und wenn einmal eine Neuanschaffung notwendig wird, so wird diese direkt durch den Chef getätigt. Dazu ist kein langwieriger Prozess mit Antragstellung bei einer Verwaltung notwendig. [...].

FD: In der Praxis hat mir bereits zu Ausbildungszeiten die enge Bindung von Patienten zum behandelnden Arzt gut gefallen. Man betreut den Patienten von der Diagnosestellung bis zur Heilung und darüber hinaus, vom Säuglings- bis zum Seniorenalter.

Da unsere Augenarztpraxis bereits seit 30 Jahren besteht, haben wir eine sehr große Patientenkartei, in der sich bis zu vier Generationen einer Familie befinden. Eine gute Organisation und die fachlich kompetente Zuarbeit der paramedizinischen Berufe haben große Bedeutung. Der Chef ist hier tatsächlich Chef, gleichzeitig Vorbild, setzt Veränderungen direkt in die Tat um und übernimmt die Verantwortung und Finanzierung für den gesamten Betrieb. In der Niederlassung ist die Schlagzahl größer als in der Klinik. Für den jungen Assistenten ist es vorteilhaft, viele Patienten zu untersuchen und so die häufigen Diagnosen sicher zu erkennen, bei ungefiltertem Patientengut.

In der Augenheilkunde wird ein Großteil des Versorgungsspektrums ambulant abgedeckt. Im ambulanten Verbund mit anderen Praxiskliniken findet sich auch für komplexe operative Eingriffe der richtige Spezialist.

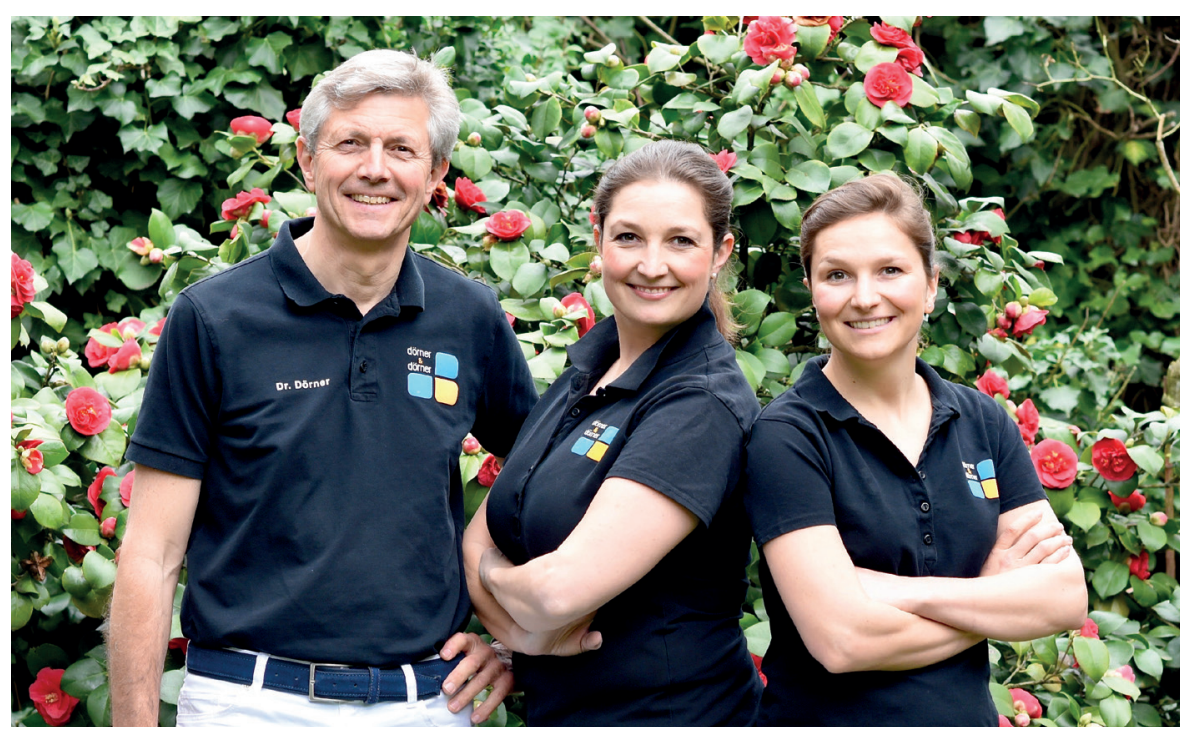

Perfektes Team: Dr. Martin Dörner, Dr. Friederike Dörner (FEBO), Johanna Dörner (v.l.n.r.). 
Als Assistenzarzt in der Klinik arbeitet man in einem großen Team und auch interdisziplinär mit anderen Fachabteilungen zusammen. Viele Entscheidungen trifft die Verwaltung und nicht der Chefarzt. Der Assistent muss hier mehr Zeit für Organisation und Dokumentation aufbringen und für das Schreiben von Briefen. Komplizierte Dienstmodelle und Urlaubspläne lassen wenig Flexibilität bei der Freizeitgestaltung.

In die Klinik kommen die meisten Patienten aufgrund einer Ein- oder Überweisung. Die erste Vorarbeit ist also bereits geleistet. Nun steht die Spezialdiagnostik und Begutachtung durch Experten, sowie die Zusammenarbeit verschiedener Fachabteilungen im Vordergrund. Der Assistenzarzt kann hier komplexe und seltene Krankheitsbilder kennenlernen. Die Behandlung extrem schwieriger Fälle schult für die Zukunft. Und auch einige Spezialfälle aus dem Nachtdienst in der Notaufnahme bleiben einem für immer in Erinnerung.

\section{Haben sich Ihre Erwartungen an die Arbeit als niedergelas- sener Augenarzt erfüllt?}

MD: Ich finde die freiberufliche ärztliche Tätigkeit jeden Tag wieder spannend. Die wunderbaren Möglichkeiten operativ wie konservativ effektiv helfen zu können, mit teilweise lange bewährten, aber auch vielen neuen, innovativen Methoden, kommen dem Patienten direkt zugute und führen zu großer Zufriedenheit bei Behandler und Patient. Die Patienten treten dem Arzt oder angehenden Facharzt entsprechend mit Respekt gegenüber.

Wenn ich die 30 Jahre Niederlassung Revue passieren lassen, dann spüre ich aber auch, dass die Furcht des frischgebackenen Facharztes, sich in eigener Praxis selbstständig zu machen, durchaus berechtigt ist.

Die Perspektive der Facharztweiterbildung ist für viele die Niederlassung. Die Dichte von bürokratischen Vorschriften, Verpflichtungen und Drohszenarien nimmt jedoch ständig zu. Für populistische Politiker ist das Gesundheitswesen nicht nur eine Spielwiese, sondern auch eine stetige Quelle von, ja man muss sagen, Arbeitsbeschaffungsmaßnahmen. Ich erinnere da nur an Zertifizierung, Datenschutz und Hygiene. Regresse und durch die eigene Standesvertretung zwangsweise eingeleitete Reglementierungen, auch mit Unterstützung der Staatsanwaltschaft (Plausibilitätsprüfung), machen die eigenverantwortliche Tätigkeit als Augenarzt nicht gerade gemütlicher. Eine repressionsfreie Standesvertretung, die gegen unsinnige, politische, bürokratische oder sonstige unlautere Bedrängung auch einmal eindeutig Stellung bezieht, würde ich mir schon manchmal wünschen. Die Reputation der Ärzteschaft wird durch Krankenkassen (Stichwort IGeL) und Politik permanent attackiert. Das ist in anderen Ländern teilweise anders, weswegen man sich nicht wundert, dass es junge, fähige Kollegen ins Ausland zieht. Die allgemein zunehmende Tendenz alles und jeden zu verklagen scheint von vielen Juristen gebilligt zu werden.

Anders als vor 30 Jahren muss der Niederlassungswillige eine Menge Geld in neue Techniken, moderne Praxisausstattung, EDV und vor allem Personal stecken, das erst angelernt werden muss zur Bewältigung eines überbordenden Papierkrieges (Beispiel IVOM).

Auch Zwangsfortbildungen - von Datenschutz über Hygiene bis zu Fortbildungspunkten - motivieren den jungen Facharzt nicht gerade.
Für die jungen Ärzte dennoch eine wichtige Erkenntnis: es wird auch in Zukunft trotz Expertensystemen und Dr. Google weiterhin nicht ohne Ärzte gehen.

\section{Was ist Ihnen an der Ausbildung wichtig? Worauf wollen Sie keinesfalls verzichten?}

JD: In der Weiterbildung muss das Gesamtpaket stimmen. Innerhalb der fünf Weiterbildungsjahre muss man eine gute Basis augenärztlicher Fachkenntnisse aufbauen. Sicherlich ist man mit Erlangen des Facharztes noch kein perfekter Diagnostiker, Therapeut oder gar Operateur; dennoch sollte es möglich sein, sich viele Skills bereits früh anzueignen und darauf aufzubauen. Dabei ist ein Ausbilder, der mir keine Frage übelnimmt, der mich für sein Fach begeistern kann, der mir etwas zutraut und mich dennoch nicht überfordert meiner Ansicht nach unbezahlbar. Und wird es einmal schwierig, so wünsche ich mir - gerade zu Beginn der Weiterbildung - die Möglichkeit, mich bei meinem Ausbilder rückversichern zu können - zur Sicherheit meiner Patienten und zu meiner eigenen Sicherheit.

Aber auch das alltägliche Arbeitsklima spielt für mich eine große Rolle. Die Arbeit muss, trotz aller Stressfaktoren und Dienstbelastungen, trotz aller Hochs und Tiefs, am Ende des Tages Spaß machen. Sichtbare Therapieerfolge und dankbare Patienten stehen dabei ganz oben auf der Prioritätenliste.

FD: Ich finde eine breite Ausbildung sehr wichtig. Ich habe davon profitiert, dass ich bei verschiedenen Weiterbildern lernen durfte. Es zeigt sich schnell, dass es mehr als einen Weg zum Ziel gibt. Es lehrt einen, Abläufe und Therapien kritisch zu hinterfragen. Medizin ist dynamisch und individuell. So sollten wir auch ausgebildet werden. Eine frühe Förderung von Begabungen, sei es operativ, organisatorisch oder als Vortragsreferent, sollte gefördert und gefordert werden. Eigenverantwortliches Lernen ist aus meiner Sicht genauso erforderlich, wie ein Ausbilder, der sich für seine Auszubildenden verantwortlich fühlt und ihren Lernfortschritt auch als seinen Erfolg wertet.

\section{Was hat Ihnen während der Ausbildung gefehlt?}

FD: Im ersten Dienst, in dem man alleine eine ganze Nacht für eine Augenklink, die Ambulanz und die Station verantwortlich ist, ist man so aufgeregt, dass man einfach nur bis zur Dienstbesprechung am nächsten Morgen um 7 Uhr überleben will.

In der kommenden Zeit wird der Schlafmangel zur Routine. Die Arbeit wird vom Tag-zum Nacht-zum Tagdienst weitergegeben. Zwischendurch kommt der Oberarzt oder der Chef. Jeder hat so viel mit seinen eigenen Aufgaben zu tun, dass für die Wertschätzung untereinander keine Zeit mehr bleibt. Jeder von uns wünscht sich Wertschätzung für seine Arbeit, egal in welchem Ausbildungsstand man gerade ist.

Viele Möglichkeiten der Weiterbildung und Fortbildung hätte ich während der Weiterbildung intensiver nutzen sollen. Ich denke da z.B. an die Möglichkeit von Fellowships, Habilitation sowie die Teilnahme an Intensivkursen und Tagungen.

Ich hatte das Glück, sehr erfahrene Ausbilder zu haben, die sich sehr für mich engagiert haben und mir somit die parallele Ausbildung 
im wöchentlichen Wechsel in Klinik und Praxis ermöglicht haben. Ich würde mir in der Augenheilkunde, einem Fach, das in der ambulanten Versorgung so viele Möglichkeiten bietet, mehr Kombinierbarkeit von Klinik- und Praxisarbeit wünschen. So kann jeder Assistent sowohl Klinikkarriere als auch Niederlassung erleben und ohne Angst auf eine Benachteiligung seine Erfahrung in allen Bereichen machen. Auch Forschungsjahre und die Möglichkeit an Kongressen und Wet Labs teilzunehmen, sollten Assistenten viel großzügiger ermöglicht werden. Dafür stehen ihnen aus meiner Sicht finanzielle Unterstützung und Fortbildungszeit zu. Im Klinikalltag sieht es leider häufig so aus, dass Fortbildungstage aus Personalmangel nicht genehmigt werden. Ein gemeinsamer Fortbildungsbesuch ist häufig nicht zu realisieren. Dienstpläne lassen keine spontanen, aber auch keine langfristigen Planungsmöglichkeiten zu.

Die Einladung oder Unterstützung zu fachlichen Vorträgen gilt als Bestechung. Eine sehr schwierige Situation für alle Beteiligten.

\section{Welche Probleme wiederholen sich bei der Weiterbildung junger Ärzte?}

MD: Junge Ärzte suchen und finden häufig Symptome frisch gelernter Krankheiten bei sich selbst. Das ist bei Augenkrankheiten nicht anders. Richtig begreifen kann man die Beschwerden der Patienten oft erst nach der selbst durchgemachten Keratitis epidemica.

Am Patienten stellt ein erstes Hemmnis der Augendiagnostik zunächst die Spaltlampe dar. Nach einem halben Jahr werden oft überraschende Details mit diesem Gerät erkannt.

Optisches Verständnis und weitere Ausbildungszeit ist erforderlich beim erfolgreichen Bedienen eines Phoropters. Ein exakter Binokularabgleich gelingt im ersten Assistenzjahr auch mit gut gemeinter Segelanleitung eher bruchstückhaft. Ein sattelfester Optiker kann in diesem Fall auch vor Absolvieren eines Schober-Kurses gute Dienste leisten. [...].

Die Praxisgemeinschaft Dörner \& Dörner ist eine große überörtliche, operative Gemeinschaftspraxis im Westmünsterland. Im Herzen der Stadt Bocholt liegt die Praxis mit ambulantem OPZentrum, welche in diesem Jahr ihr 30-jähriges Jubiläum feiert. Die Praxis hat eine moderne, hochtechnische Ausstattung, um ein breites Spektrum der ambulanten Versorgung im großen Einzugsgebiet abzudecken. Seit drei Jahrzehnten bildet Dr. Martin Dörner medizinische Fachangestellte und Assistenzärzte im Bereich der konservativen und operativen Augenheilkunde aus. Das Team wird ergänzt durch Augenoptikermeister und Refrak tivmanager, die insbesondere im Bereich Refraktivsprechstunde und Kontaktlinsenversorgung ihre Expertise beitragen. Ebenfalls im Haus in der Königstraße befindet sich die chirurgische Gemeinschaftspraxis von Dres. Dörner, Dehn und Bongers. Seit dem Frühjahr 2018 ist der Standort Neheim im Sauerland zur Gemeinschaftspraxis der Augenheilkunde dazugekommen.

\section{Johanna Dörner}

2010-2016 Studium der Humanmedizin an der Westfälischen Wilhelms-Universität Münster

2013/2014 Auslandssemester an der RSU Riga, Lettland

$2017 \quad$ Weiterbildung in der Allgemeinchirurgie

2017 Doktorandin im Experimental Eye Research Institute Bochum-Langendreer unter Leitung von PD Dr. Stephanie Joachim

2018 Weiterbildung in der Augenheilkunde

\section{Dr. Friederike Dörner (FEBO)}

2000 Europameisterin der Pony-Vielseitigkeitsreiterei

2011 Staatsexamen Humanmedizin an der HeinrichHeine-Universität Düsseldorf

2011 Promotion an der Uniklinik RWTH Aachen

2017 Fachärztin der Ophthalmologie und FEBO (FellowEuropean Board ofOphthalmology)

2018 Niederlassung mit eigenem KV-Sitz Neheim/ Arnsberg, Partnerschaft in der Gemeinschaftspraxis Dörner \& Dörner

\section{Dr. Martin Dörner}

1974 Studium der Tiermedizin, tierärztliche Hochschule Hannover

1976 Studium der Humanmedizin in Erlangen und Köln

1981 Fliegerarzt i.V. Marinefliegergeschwader 1

1982 Uniklinik Köln, Zentrum für Augenheilkunde

1986 Promotion bei Prof. Heimann

1988 Niederlassung; Ambulante Mikrochirurgie Bocholt

2000 Weiterbildungsberechtigung für 36 Monate.

Die Weiterbildung in der Praxis wird durch die mindestens 2-jährige Klinikzeit ergänzt. Wir freuen uns, wenn unsere Schützlinge danach den Weg zu uns zurückfinden. Oft haben die frisch gebackenen Fachärzte aber schon andere Pläne und Angebote. Leider möchten viele nicht an die alte Ausbildungsstätte zurück.

Aber auch nach der Facharztprüfung lernt man in der Praxis noch immer weiter. Auch als Ausbilder. Das macht das Fach auch so spannend.

\section{Die Facharztweiterbildung ist eine herausfordernde Zeit und dann noch als Familie zusammenzuarbeiten... Wo gibt bzw. gab es hier das größte Konfliktpotential?}

JD: Die Zusammenarbeit funktioniert in unserer Familie erstaunlich konfliktfrei. Wir sind im Gemeinschaftspraxisumfeld auf- und zusammengewachsen und haben uns immer schon mit Rat und Tat zur Seite gestanden. Wir kennen die Stärken und Schwächen der anderen und wissen damit prima umzugehen.

FD: Konfliktpotential ist überall vorhanden, ob in der Klinik oder in der Familie. Ich könnte mir dennoch keinen besseren Ausbilder als meinen Vater vorstellen. Seine Hoffnung, mich als Praxispartner und Nachfolger zu gewinnen, hat sich erfüllt. Als Praxispartner 
haben wir unterschiedliche Stärken und Schwächen. Stärken nutzen wir für Optimierungen. An Schwachpunkten greifen wir ein $\neg$ ander unter die Arme. Wir ziehen an einem Strang.

Die Hemmungen innerhalb der Familie nach Zweitmeinungen zu fragen sind gering, die Patientenbindung ist familiär, die Flexibilität groß. All das macht unseren Familienbetrieb zu einem angenehmen Arbeitsumfeld.

Friederike Dörner, Sie sind seit Anfang 2017 fertig mit Ihrer Facharztweiterbildung - wie ging Ihr Weg danach weiter?

FD: Zunächst habe ich als angestellter Facharzt bei meinem Vater in der Praxis gearbeitet. Ich hatte dann das Glück, einen KV Sitz in Neheim/Arnsberg im Sauerland zugesprochen zu bekommen, sodass ich heute als Praxispartner meine eigene Praxis als Nebenbetriebsstätte habe und weiterhin im ambulanten OP-Zentrum der Gemeinschaftspraxis tätig sein kann.

\section{Johanna Dörner, Ihre Weiterbildung hat gerade erst begon- nen - gibt es schon Zukunftspläne? \\ JD: Meine Dissertation möchte ich in Kürze fertigstellen. Bis zum Jahresende werde ich in der Praxis arbeiten und mich zum Jahres- wechsel bei einer Augenklinik mit voller Weiterbildungsberechti- gung bewerben. Einige Favoriten habe ich zwar schon im Kopf, aber ich bin noch nicht ganz sicher, wo ich mich bewerben werde. Ich bin schon gespannt auf das neue Arbeitsumfeld, die neuen Kol- legen, auf die Chancen und Herausforderungen der Klinik.}

Was möchten Sie den Weiterbildungsassistenten - neben den fachlichen Fähigkeiten - gerne mit auf den Weg geben?

MD: Fachliche Fähigkeiten sind eine Grundvoraussetzung. Menschliche Fähigkeiten sind zur erfolgreichen Ausübung der ärztlichen Tätigkeit ebenso wichtig.
Das Zentrum unserer Bemühungen muss immer der Patient sein. Der initiale Blickkontakt mit dem Kranken, verbunden mit einer offenen Frage nach seinem Befinden und dass man inm überbringt, dass man nun Zeit nur für ihn hat: Das ist wichtig. Weniger wichtig sind Displays mit Patientendaten oder administrativer Schnickschnack. Wenn ich dem Patienten eine Therapie empfehle, würde ich sie genau auch so bei mir selbst anwenden. Ich versuche, den Assistenten mit gutem Beispiel voranzugehen und ihnen zu zeigen, dass ich mir einen Patientenstamm und auch den verdienten Respekt erarbeiten kann. Gegenseitiger Respekt verbessert die Compliance. Und Respekt vor dem Auge als sensibles Organ ist der Grundstein für eine erfüllende und erfolgreiche Tätigkeit.

Kontaktadresse: Laser-24 GmbH, Königstraße 34, 46397 Bocholt, Deutschland

Ungekürztes Originalinterview: «Weiterbildung in der Niederlassung: Eindrücke aus einer Arztpraxis für Augenheilkunde», ein Beitrag auf www.operation-karriere.de (https://www.operation-karriere. de/karriereweg/assistenzarzt/weiterbildung-in-der-niederlassung-eindruecke-aus-einer-arztpraxis-fuer-augenheilkunde.html)

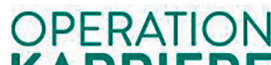

Aktuelle Studienlage

\section{Laufende klinische Studien zu Kombinationstherapien des Melanoms}

\begin{tabular}{|c|c|c|c|c|}
\hline Targets & Trial Number & Intervention & Disease & Trial Phase \\
\hline \multirow[t]{4}{*}{ MDSC } & NCT03200847 & ATRA (Vesanoid)+pembrolizumab & Advanced melanoma & I, II \\
\hline & NCT02403778 & ATRA+ipilimumab & Advanced melanoma & $\|$ \\
\hline & NCT03161431 & $\begin{array}{l}\text { SX-682 alone or in combinationwith } \\
\text { pembrolizumab }\end{array}$ & Melanoma (III, IV) & । \\
\hline & NCT02259231 & $\begin{array}{l}\text { RTA } 408 \text { (Omaveloxolone)+nivolumab or } \\
\text { ipilimumab }\end{array}$ & Unrespectable or metastaticmelanoma & $\mid \mathrm{b}, \mathrm{|l}$ \\
\hline CAF & NCT03875079 & RO6874281+pembrolizumab & Metastatic melanoma & $\mathrm{lb}$ \\
\hline TAM & NCT01363206 & $\begin{array}{l}\text { GM-CSF (Leukine,Sargramostim)+without } \\
\text { ipilimumab }\end{array}$ & Unresectable metastaticmelanoma & $\|$ \\
\hline \multirow[t]{2}{*}{ Treg } & NCT02203604 & Aldesleukin (IL-2)+ipilimumab & Metastatic melanoma (IIIA-IV) & $\|$ \\
\hline & NCT02983045 & $\begin{array}{l}\text { NKTR-214 (PEGylated IL-2)+nivolumab } \\
\text { with or without ipilimumab }\end{array}$ & $\begin{array}{l}\text { Advanced malignancies,including } \\
\text { melanoma }\end{array}$ & l, II \\
\hline
\end{tabular}




\begin{tabular}{|c|c|c|c|c|}
\hline Targets & Trial Number & Intervention & Disease & Trial Phase \\
\hline & NCT03548467 & NKTR-214 after prior anti-PD-1 therapy & $\begin{array}{l}\text { Advanced malignancies,including me- } \\
\text { lanoma }\end{array}$ & I, II \\
\hline & NCT03635983 & NKTR-214+nivolumab ornivolumab alone & $\begin{array}{l}\text { Untreated, inoperable ormetastatic } \\
\text { melanoma }\end{array}$ & III \\
\hline & NCT03138889 & NKTR-214+pembrolizumab & $\begin{array}{l}\text { Advanced malignancies,including me- } \\
\text { lanoma }\end{array}$ & I, II \\
\hline & NCT03435640 & $\begin{array}{l}\text { Intratumoral NKTR-262+systemic NKTR- } \\
214 \text { with orwithout nivolumab }\end{array}$ & Melanoma and other cancertypes & I, II \\
\hline & NCT03635983 & NKTR-214+nivolumab ornivolumab alone & $\begin{array}{l}\text { Untreated, inoperable ormetastatic } \\
\text { melanoma }\end{array}$ & III \\
\hline \multirow[t]{4}{*}{ Microbiome } & NCT03341143 & $\begin{array}{l}\text { Fecal microbiota } \\
\text { transplant(FMT)+pembrolizumab }\end{array}$ & $\begin{array}{l}\text { Advanced melanoma patients,non- } \\
\text { responders }\end{array}$ & ॥ \\
\hline & NCT03817125 & $\begin{array}{l}\text { Vancomycin or placebopretreatment+ } \\
\text { nivolumab+SER-401 or placebo }\end{array}$ & Unresectable or metastaticmelanoma & $\mathrm{lb}$ \\
\hline & NCT03772899 & $\begin{array}{l}\text { FMT for a healthy do- } \\
\text { nor a weekbefore approved } \\
\text { melanomatreatment(pembrolizumab/ } \\
\text { nivolumab) }\end{array}$ & Advanced melanoma & । \\
\hline & NCT03643289 & $\begin{array}{l}\text { Comparison of gut microbiomebefore and } \\
\text { during anti-PD-1therapy (till week 9) }\end{array}$ & Advanced melanoma stage IV & Observational \\
\hline \multirow[t]{2}{*}{ Hypoxia } & NCT03311308 & $\begin{array}{l}\text { Metformin+pembrolizumab orpembroli- } \\
\text { zumab alone }\end{array}$ & $\begin{array}{l}\text { Advanced, unresectablemelanoma stage } \\
\text { III or IV }\end{array}$ & । \\
\hline & NCT03171064 & Exercise+nivolumab orpembrolizumab & Metastatic melanoma & $\|$ \\
\hline \multirow[t]{14}{*}{ Tumor cells } & NCT02799901 & $\begin{array}{l}\text { Hypofractionated radiationtherapy (RT) } \\
\text { (27 Gy over 3fractions)+nivolumab }\end{array}$ & Advanced melanoma & $\|$ \\
\hline & NCT03693014 & $\begin{array}{l}\text { Hypofractionated RT+Ipilimumab, Nivo- } \\
\text { lumab orPembrolizumab, continuedaccor- } \\
\text { ding to the standardschedule }\end{array}$ & Metastatic cancer, includingmelanoma & ॥ \\
\hline & NCT02406183 & Ipilimumab+RT & Metastatic melanoma & । \\
\hline & NCT04042506 & Nivolumab+RT & Metastatic melanoma & $\|$ \\
\hline & NCT04017897 & $\begin{array}{l}\text { Anti-PD1 (pembrolizumab } \\
\text { ornivolumab)+RT }\end{array}$ & $\begin{array}{l}\text { Unresectable, naive } \\
\text { metastaticmelanoma(IIIB to IVM1c) }\end{array}$ & ॥ \\
\hline & NCT01449279 & Ipilimumab+RT & Metastatic melanoma & $\|$ \\
\hline & NCT01689974 & Ipilimumab+RT or ipilimumabalone & Metastatic melanoma & $\|$ \\
\hline & NCT01769222 & Ipilimumab+RT or ipilimumabalone & $\begin{array}{l}\text { Recurrent malignancies,including } \\
\text { melanoma }\end{array}$ & I, II \\
\hline & NCT02659540 & $\begin{array}{l}\text { Nivolumab+ipilimumab incombination } \\
\text { with conventionalor hypofractionated RT }\end{array}$ & Unresectable melanoma stage IV & । \\
\hline & NCT02263508 & Pembrolizumab+T-VEC orplacebo & Stage IIIB-IVM1c melanoma & III \\
\hline & NCT04068181 & $\begin{array}{l}\text { Pembrolizumab+T-VEC afterprogression } \\
\text { on anti-PD-1therapy }\end{array}$ & Stage IIIB-IVM1d melanoma & $\|$ \\
\hline & NCT01740297 & Ipilimumab+T-VEC oripilimumab alone & Stage IIIB-IV metastaticmelanoma & I, II \\
\hline & NCT02965716 & Pembrolizumab+T-VEC & Stage IIIB-IV metastaticmelanoma & $\|$ \\
\hline & NCT03842943 & Neoadjuvant pembrolizumab+T-VEC & Resectable stage 3 melanoma & $\|$ \\
\hline \multirow[t]{2}{*}{ Tumormutations } & NCT02902042 & Encorafenib+binimetinib+pembrolizumab & Metastatic BRAF V600 mutantmelanoma & I, II \\
\hline & NCT02910700 & $\begin{array}{l}\text { Nivolumab+trametinib with orwithout } \\
\text { dabrafenib }\end{array}$ & $\begin{array}{l}\text { BRAF-mutated or wild typemetastatic } \\
\text { stage III-IVmelanoma }\end{array}$ & $\|$ \\
\hline
\end{tabular}




\begin{tabular}{|c|c|c|c|c|}
\hline Targets & Trial Number & Intervention & Disease & Trial Phase \\
\hline & NCT02908672 & $\begin{array}{l}\text { Cobimetinib+vemurafenibwith atezoli- } \\
\text { zumab or placebo }\end{array}$ & Metastatic BRAF V600 mutantmelanoma & III \\
\hline & NCT02303951 & Vemurafenib+cobimetinib+atezolizumab & BRAF V600 mutant stage IIIC-IVmelanoma & $\|$ \\
\hline & NCT01767454 & $\begin{array}{l}\text { Dabrafenib+ipilimumab ordabrafenib+tra } \\
\text { metinib+ipilimumab }\end{array}$ & $\begin{array}{l}\text { Metastatic or unresectable BRAFV600 } \\
\text { mutant melanoma }\end{array}$ & । \\
\hline \multirow[t]{2}{*}{$\begin{array}{l}\text { Epigenetic } \\
\text { modifications }\end{array}$} & NCT03765229 & Entinostat+pembrolizumab & Stage III-IV metastaticmelanoma & $\|$ \\
\hline & NCT02437136 & Entinostat+pembrolizumab & $\begin{array}{l}\text { Advanced malignancies,including } \\
\text { melanoma }\end{array}$ & $|b| \mid$, \\
\hline \multirow[t]{6}{*}{ Neoantigens } & NCT03929029 & $\begin{array}{l}\text { NeoVax+Montanide }{ }^{\oplus} \text { withnivolumab+ipil } \\
\text { imumab }\end{array}$ & Advanced melanoma & IB \\
\hline & NCT02385669 & Peptide Vaccine+Ipilimumab & $\begin{array}{l}\text { Stage IIA-IV melanoma(advanced, } \\
\text { adjuvant,neoadjuvant) }\end{array}$ & I, II \\
\hline & NCT03047928 & PD-L1/IDO peptide vaccine+nivolumab & Metastatic melanoma & I, II \\
\hline & NCT03633110 & $\begin{array}{l}\text { GEN-009 Adjuvant } \\
\text { Vaccine+pembrolizumab or nivolumab }\end{array}$ & Solid tumors, includingmelanoma & I, II \\
\hline & NCT04072900 & $\begin{array}{l}\text { Personalized neoantgen } \\
\text { peptidevaccine+anti-PD-1+rhGM- } \\
\text { CSF+Imiquimod 5\%Topical Cream }\end{array}$ & Metastatic melanoma & । \\
\hline & NCT04091750 & $\begin{array}{l}\text { Nivolumab+ipilimumab+cabozantinib } \\
\text { followed bynivolumab+cabozantinib }\end{array}$ & Advanced melanoma & $\|$ \\
\hline \multirow[t]{10}{*}{$\begin{array}{l}\text { Other immune } \\
\text { checkpoint } \\
\text { molecules }\end{array}$} & NCT02676869 & IMP321+pembrolizumab & $\begin{array}{l}\text { IMP321+pembrolizumabStage III-IV } \\
\text { advancedmelanoma }\end{array}$ & । \\
\hline & NCT02519322 & $\begin{array}{l}\text { Nivolumab+relatimab or+ipilimumab or } \\
\text { alone beforesurgery }\end{array}$ & Stage IIIb-IV advancedmelanoma & $\|$ \\
\hline & NCT03743766 & $\begin{array}{l}\text { Relatimab+nivolumab or eachdrug alone } \\
\text { followed byrelatimab+nivolumab in } \\
\text { allsubjects }\end{array}$ & Unresectable or metastaticmelanoma & $\|$ \\
\hline & NCT03470922 & Relatimab+nivolumab ornivolumab alone & Unresectable or metastaticmelanoma & $\|\|$, \\
\hline & NCT03652077 & $\begin{array}{l}\text { NCAGN02390 antibody againstTIM-3 } \\
\text { alone }\end{array}$ & $\begin{array}{l}\text { Advanced malignancies,including } \\
\text { melanoma }\end{array}$ & । \\
\hline & NCT04139902 & $\begin{array}{l}\text { Neoadjuvant therapy with PD-1 inhibitor } \\
\text { dostarlimab (TSR-042)or dostarlimab } \\
\text { (TSR-042)+TSR-022 (TIM-3 inhibitor) }\end{array}$ & Stage IIIB-IVadvanced melanoma & $\|$ \\
\hline & NCT03708328 & $\begin{array}{l}\text { RO7121661, bispecific anti-PD-1and } \\
\text { anti-TIM-3 antibody }\end{array}$ & $\begin{array}{l}\text { Advanced malignancies,including } \\
\text { melanoma }\end{array}$ & । \\
\hline & NCT02817633 & $\begin{array}{l}\text { TSR-022 (anti-TIM-3) alone or+TSR-042 } \\
\text { (anti-PD-1) or triplecombination of TSR- } \\
\text { 022(anti-TIM-3), TSR-042(anti-PD-1) and } \\
\text { TSR-033(anti-LAG3) }\end{array}$ & $\begin{array}{l}\text { Advanced malignancies, including } \\
\text { melanoma }\end{array}$ & I \\
\hline & NCT03628677 & $\begin{array}{l}\text { AB154 (anti-TIGIT) alone or+AB122 } \\
\text { (anti-PD-1) }\end{array}$ & $\begin{array}{l}\text { Advanced malignancies, including } \\
\text { melanoma }\end{array}$ & \\
\hline & NCT03119428 & $\begin{array}{l}\text { OMP-313M32 (anti-TIGIT) } \\
\text { aloneor+nivolumab }\end{array}$ & $\begin{array}{l}\text { Advanced malignancies,including } \\
\text { melanoma }\end{array}$ & । \\
\hline
\end{tabular}

Aus: Petrova V, Arkhypov I, Weber R, Groth C, Altevogt P, Utikal J, Umansky V.: Modern Aspects of Immunotherapy with Checkpoint Inhibitors in Melanoma. Int J Mol Sci. 2020 Apr; 21(7): 2367. 